\title{
Multiple Input Multiple Output with Antenna Number Modulation and Adaptive Antenna Selection
}

\author{
JEHAD M. HAMAMREH ${ }^{1}$, MUHAMMET KIRIK ${ }^{1}$, MEHMET O. SAGMAN ${ }^{1}$, NAOKI ISHIKAWA ${ }^{2}$ \\ ${ }^{1}$ J. M. Hamamreh, M. Kirik and M. O. Sagman are with WISLAB-TELENG for Wireless Research at the Department of Electrical-Electronics Engineering, \\ Antalya Bilim University, Antalya, Turkey. (web: https://sites.google.com/view/wislab // email: jehad.hamamreh@antalya.edu.tr). \\ ${ }^{2} \mathrm{~N}$. Ishikawa is with the Graduate School of Information Sciences, Yokohama national University, Tokyo, Japan (e-mail: ishikawa-naoki-fr@ynu.ac.jp) \\ Corresponding author: Jehad M. Hamamreh (e-mail: jehad.hamamreh@gmail.com).
}

The Matlab simulation codes used to generate the results in this paper can be found at https://researcherstore.com with the name MIMO-ANM. This research was partly funded by TUBITAK under Grant/Award Number 119E392.

\begin{abstract}
Multiple input multiple output system with antenna number modulation (MIMO-ANM) is proposed in this paper as a novel transmission method that can be used instead of conventional MIMO with spatial modulation (MIMO-SM) for providing more advantageous and reliable data transmission in future wireless systems. In this scheme, the additional data bits are conveyed by changing the number of active transmit antennas instead of their indices while the number of receive antennas is kept singular. As such, MIMO-ANM is different from MIMO-SM as it can make the selection of the antennas in MIMO to be both channel and data dependent at the same time, resulting in what is called MIMO-ANM with adaptive antenna selection (MIMO-ANM-AAS), while MIMO-SM is unable to make the selection of the antennas channel-dependent because the indices of the selected antenna are purely data-dependent. By using such a transmission scheme, the overall reliability of the transmitted data stream can significantly be improved, which characterizes MIMO-ANM-AAS with much lower bit errors compared to MIMO-SM. In this paper, the concepts of MIMO-ANM and MIMO-ANM-AAS are established on the basis of conventional MIMO using binary phase shift keying (BPSK) symbol modulation over a Rayleigh fading channel. The validity of the system is investigated, where analytical and simulation results showing the bit error rate (BER) performance of the system are given. The numerical computer simulations furthermore compare the performances of MIMO-ANM-AAS with MIMO-SM to show its superiority, and the advantages of the concept are discussed. The acquired results show that MIMO-ANM-AAS offers highly reliable and resilient system thanks to its simultaneous bit transmission by different number of active antennas that can be made adaptive to the channel gains.
\end{abstract}

INDEX TERMS MIMO, MIMO-ANM, antenna number modulation, adaptive antenna selection, spatial modulation, Rayleigh Channel, BER, Wireless Communication, 6G.

\section{INTRODUCTION}

The growing requirements that come with the continuous development of technology, the increase of demand of society on Internet services and applications, and the increasing $5 \mathrm{G}$ and beyond use cases necessitate the addressing of key performance metrics, such as data transmission rate, power efficiency and reliability of the conveyed data, and how they can be implemented in a more highly optimized, efficient, and beneficial manner [1], [2].

Technologies such as OFDM, MIMO, NOMA, etc. have constantly tried to fulfill these requirements. In particular,
MIMO has become one of the most rooted techniques due to its great advantages that manifested during its early development in the 1980s and 1990s [3]-[5]. Even though MIMO has become an inevitable method to provide enviable data rates and high quality, it still leaves an open door for the researchers to improve the efficiency of MIMO transmission systems [6].

Although MIMO systems were partially able to meet some of these requirements, they have introduced their own problems and complexities. The main problem of MIMO that has to be overcome is its transceiver complexity. As 
the number of transmit and receive antennas increases, the complexity increases as well. Another major issue is the inter-channel interference (ICI) that emerges because of the simultaneous multi-antenna transmission. To eliminate these effects (or at least keep them at a minimum level) from one hand, and maximize the efficiency of MIMO systems from another hand, different MIMO techniques have been proposed throughout the years. One of these techniques is what is called "MIMO with Spatial Modulation" [7], which utilizes the indices of multiple antennas as a new dimension to send extra information bits besides those sent by conventional modulation schemes, such as M-PSK and M-QAM.

In recent years, there has been a huge interest in developing new improved modulation schemes that can enhance the system performance in terms of spectral and power efficiency. These schemes and techniques, which are exclusively surveyed in [8]-[10], are basically derived from index modulation concept which can convey additional information bits besides those carried by conventional QAM-modulated symbols through utilizing the indices of multiple entities (i.e., time, space, frequency, cote, etc) as a third dimension for conveying additional information bits.

However, spatial modulation technique is still impeded by many disadvantages that impact the system efficiency and its reliability. Particularly, this technique along with all other schemes based on it or similar to it, which were comprehensively discussed and explained in [8]-[12], suffer from the inability to make the selection of the antennas involved in the index modulation process to be channel-dependent as the selected antennas indices are purely data-dependent. This inherent problem occurs because of the fact that we cannot make the selection of the antenna in spatial modulation-based schemes to be both channel and data dependent simultaneously at the same time [11]. Thus, this results in sacrificing the possible performance gain (coding and/or diversity gain) that could result from channel-adaptive MIMO transmission schemes such as transmit antenna selection or subset antenna selection methods [13], [14].

In this paper, a novel method called Multiple Input Multiple Output with Antenna Number Modulation (MIMO-ANM) is proposed for addressing the above mentioned drawbacks of spatial modulation schemes. In this method, the additional data bits are sent by changing the number (instead of indices) of transmit antennas in usage. By letting a portion of the incoming bits determine the number of transmit antennas to be used, the rest of the incoming bits can then be transmitted from the respective active antennas by using conventional PSK/QAM modulation. After presenting and explaining the basic design concept of MIMO-ANM, we show how the proposed scheme can solve the aforementioned problems related to index modulation scheme by proposing and developing a novel enhanced version of MIMO-ANM scheme that is capable of making the selection of the antennas in MIMO to be both channel and data dependent simultaneously. The resulting channel-based adaptive scheme is coined and called as MIMO-ANM with adaptive antenna selection (MIMO-
ANM-AAS). Such a novel transmission strategy results in sending additional information by the number of antennas that are activated at each transmission slot, making the selection of antennas to be channel dependent (like channel-based antenna selection methods in MIMO system). Consequently, the proposed scheme is expected to concurrently enhance reliability and secrecy, making it a good candidate for future $5 \mathrm{G}$ and beyond wireless networks.

It should be pointed out that the proposed MIMO-ANM concept in this work is inspired from our previously conducted research on OFDM with subcarrier number modulation (OFDM-SNM), which has recently been introduced in [15], [16]. The proposed MIMO-ANM has a completely different system model design along with different challenges and analysis procedure. This is so because the number of antennas are considered as the data-conveying feature in MIMO-ANM scheme rather than the number of subcarriers as is the case in OFDM-SNM systems.

The remaining parts of this paper are organized as follows. In section II, the system model of the MIMO-ANM concept is comprehensively explained and all the details are specified. In section III, the concept of MIMO-ANM-AAS is introduced and discussed. In section IV, the performance analysis of the system is performed. In section $\mathrm{V}$, the simulation results are exhibited and explained. Lastly, part VI concludes the paper ${ }^{1}$.

\section{SYSTEM MODEL OF MIMO-ANM}

In this study, a single-user point-to-point communication link operating under a Rayleigh fading channel is considered. The transmitter is equipped with $T$ number of antennas, whereas the receiver is using $R$ number of antennas. For simplicity in the presentation of the proposed concept, the case when $R=1$ is considered in this study, with the note that extending the system model to general case of any $R$ is straight forward.

The new proposed transmission technique named as Multiple Input Multiple Output with Antenna Number Modulation (MIMO-ANM) basically manipulates the number of transmit antennas at the transmitter to send more data bits per antenna, besides those bits that are usually transmitted by conventional PSK/QAM modulation. This would result in forming a new dimension for modulation called number of antennas in the spatial domain. This eventually yields a 3D modulation scheme that exploits not only the in-phase and quadrature components of the signal constellation diagram for sending information bits, but also the number of antennas to send extra bits.

\section{A. THE TRANSMITTER SIDE DESIGN OF MIMO-ANM}

The general structure of the proposed MIMO-ANM transmitter is shown in Fig. 1. For a given data stream of incoming bits, two sub-streams of bits are formed by grouping the bits from the end and beginning sides of the stream. The

\footnotetext{
${ }^{1}$ Notations: We denote vectors by bold-small letters, matrices by boldcapital letters, scalar numbers by non-bold capital letters, and complex numbers or elements indexing by non-bold small letters based on the context.
} 


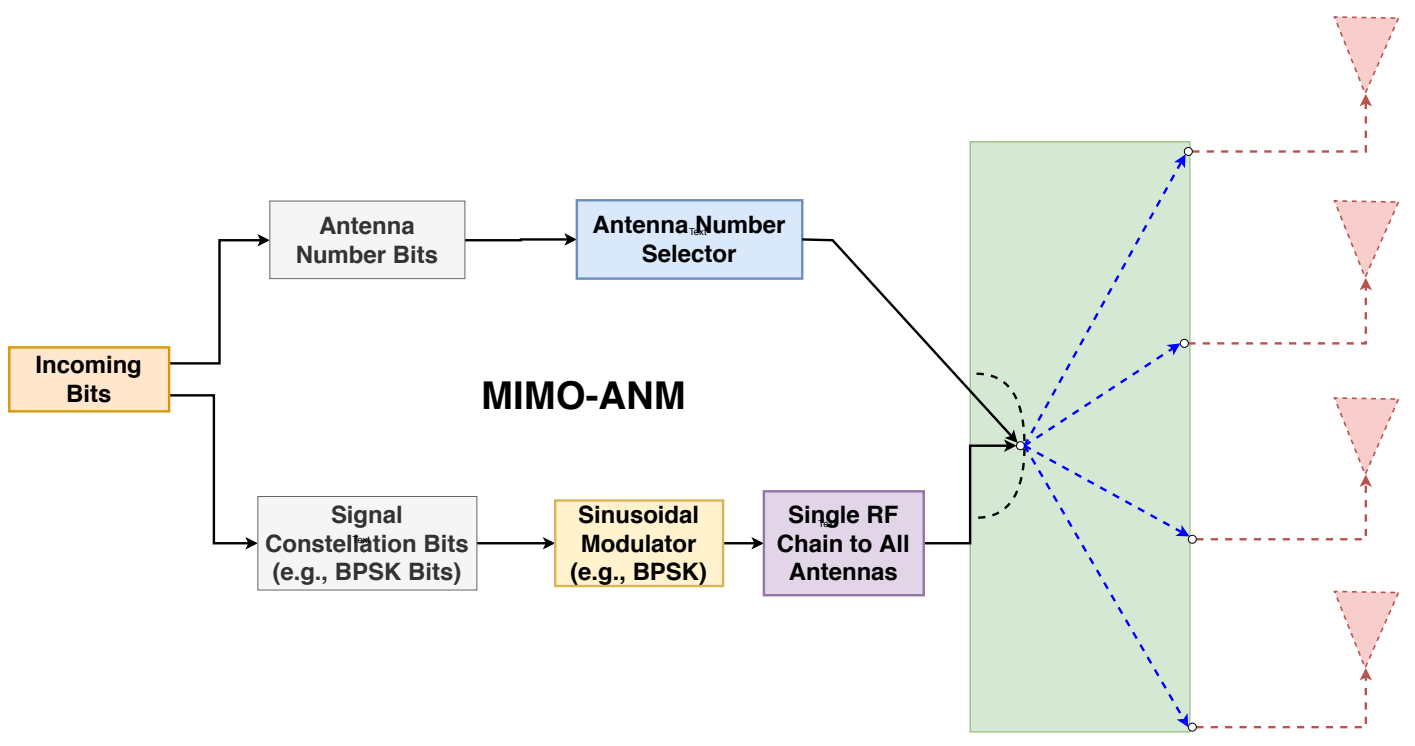

FIGURE 1. Transmitter structure of MIMO-ANM.

bits' group coming from the end side of the stream will be mapped to antennas number activation patters, and thus called as "ANM Bits", whereas the bits group coming from the beginning side of the stream will be mapped to signal constellation points, and thus named as "Main Bits". In other words, the bits at the end side of the data steam are decoupled from the main data stream and named as "ANM Bits", whereas the bits at the beginning side of the data stream are called as "Main Bits".

Accordingly, after a data stream of total number of bits $N=N_{1}+N_{2}$ is separated into the Main bits $\left(N_{1}\right)$, which is determined by the signal constellation modulation order $M$ as per this formula $N_{1}=\log _{2}(M)$, and the ANM Bits $\left(N_{2}\right)$, which is determined by the number of available transmit antennas $T$ as per this formula $N_{2}=\log _{2}(T)$, the main bits are modulated by conventional BPSK, whereas the ANM bits are used to modulate the number of antennas through using a mapping or lookup table that maps ANM bits' groups into specific number of active antennas. For the special case where the total number of antennas available for transmission is four (i.e., $T=4$ ), the mapping process is given by Table I. To clarify this process, it can be said that, if the ANM bits group or couple is "00", then the number of active antennas is one; if ANM bits group is "01", then the number of active antennas is two; if ANM bits group is "10", then the number of active antennas is three; and if ANM bits group is "11", then the number of active antennas is four. The mapping numbers of each antenna is defined by the number of antennas in the system. If $T=2^{N_{2}}$ is assumed as the number of the transmit antennas in the system, where $N_{2}$ represents the number of data bits for each ANM group. For example, for eight transmit antennas $T=2^{N_{2}}=8$, $N_{2}=\log _{2}(T)=3$, then the possible ANM bits are grouped in three bits from "000" till "111". In order to be able to compare MIMO-ANM with the other recognized schemes in
TABLE 1. ANM mapper with $N_{2}=2$ bits \& $T=4$

\begin{tabular}{|c|c|}
\hline ANM bits $\left(N_{2}\right)$ & Active Antennas Pattern (v) \\
\hline$\left[\begin{array}{ll}0 & 0\end{array}\right]$ & {$\left[\begin{array}{llll}1 & 0 & 0 & 0\end{array}\right]$} \\
\hline$\left[\begin{array}{ll}0 & 1\end{array}\right]$ & {$\left[\begin{array}{llll}1 & 1 & 0 & 0\end{array}\right]$} \\
\hline$\left[\begin{array}{ll}1 & 0\end{array}\right]$ & {$\left[\begin{array}{llll}1 & 1 & 1 & 0\end{array}\right]$} \\
\hline$\left[\begin{array}{ll}1 & 1\end{array}\right]$ & {$\left[\begin{array}{llll}1 & 1 & 1 & 1\end{array}\right]$} \\
\hline
\end{tabular}

the literature, and simplify its description, the case composed of four transmit antennas $(T=4)$ and one receive antenna $(R=1)$ is considered in the description of the proposed scheme throughout the coming sections of this paper.

Now after performing both the ANM modulation process and signal constellation modulation (e.g., BPSK) process, each individual formed complex data symbol $(x)$, which is basically the transmitted M-ary PSK/QAM symbol corresponding to the Main bits, is sent from the all the active antennas, which are determined by Table I, over the channel. Then, the transmitted data symbols get affected (multiplied) by the flat fading channel coefficients corresponding to the active antennas in the system. The signal reaching at the receiver $(y)$ is then affected by the noise $w$ with zero mean and $N_{0}$ variance at the receive antenna. Thus, the transceiver $1 \times 4$ MISO system model formula of this concept per symbol level $^{2}$ is given as

$$
y=\sqrt{\frac{P}{V}} \times \mathbf{h} \times \mathbf{v} \times x+w,
$$

where $P$ is the transmit power per data symbol, and $V=$ $\|\mathbf{v}\|_{2}=\sum_{i=1}^{T} v_{i}^{2}$ is the total number of active antennas selected for transmission out of $T$ available antennas in each

\footnotetext{
${ }^{2}$ Note that we consider symbol level-based system modeling in our study instead of block-based system modeling in order to simplify the mathematical treatments of the proposed scheme from one aspect and to enable finding the exact forms effective channel fading distributions per symbol, which will enable deriving closed form expression for the symbol and bit error rates.
} 
channel use. The flat fading channel vector is represented by $\mathbf{h}=\left[h_{11}, h_{12}, h_{13}, h_{14}\right] \in \mathbb{C}^{1 \times 4}$ in which the element represents a circularly symmetric complex Gaussian channel coefficient with zero mean and unity variance, corresponding to the response between $R^{t h}$ receive antenna and $T^{\text {th }}$ transmit antenna, whereas $\mathbf{v} \in \mathbb{R}^{4 \times 1}$ is the activation pattern vector composed of zeros for inactive antennas and ones for active antennas, which are determined according to $N_{2}$ bits and the mapping/lookup process given in Table I.

By using the antenna number feature of the MIMO system, the additional data bits are sent without using extra spectra or power. Also, the mapping process is completely independent of the indices or positions of the antennas, which is one of the key differences from spatial index modulation schemes that cannot make the selection of the active antennas to be both data and channel dependent simultaneously, whereas MIMOANM can seamlessly achieve that as will be explained in the next section.

Example: To exemplify the whole mapping procedure in MIMO-ANM modulation, the following example is given and explained. Assume the incoming bit sequence is given as "100011011" in a MIMO system, which consists of four transmit antennas $(T=4)$ and one receive antenna $(R=1)$. The number of different ANM groups can be identified as $2^{N_{2}}=T=4$, where the number of bits per group is $N_{2}=\log _{2}(T)=2$. This means that we have four groups of two bits each, where each of these groups determines how many antennas are activated. This will be used for the transmission of each respective bit from the ANM Bits stream. The separation of the incoming data bits into Main Bits (which is modulated by BPSK) and ANM Bits (which is modulated by the number of antennas) for this example is shown in Fig. 2.

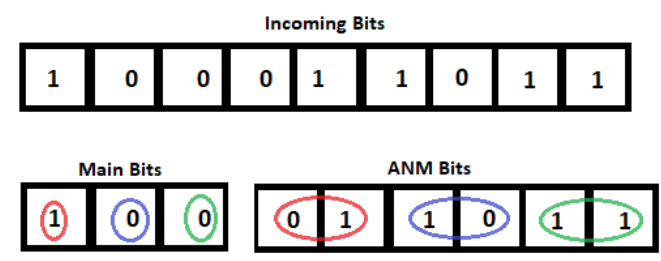

FIGURE 2. Main Bits and ANM Bits Separation from Incoming Data Stream for 4 Transmit Antennas and 1 Receiver Antenna when BPSK is used.

After this step, the MIMO-ANM process will officially begin. Since the first couple of ANM bits are "01" and it corresponds to the activation of two antennas, the first bit of the Main Bits sequence, which is "1", will be transmitted simultaneously by two antennas as demonstrated by the red lines in Fig. 3. For the next couple, "10" will correspond to the activation of three antennas. Thus, the second bit from the Main Bits sequence, which is "0" will be transmitted from three antennas. Lastly, the last couple of the ANM Bits "11" corresponds to four antennas, which means the transmission of the last bit of the Main Bits sequence, which is "0", will be done from four different antennas. This is visually displayed by Fig. 3 .

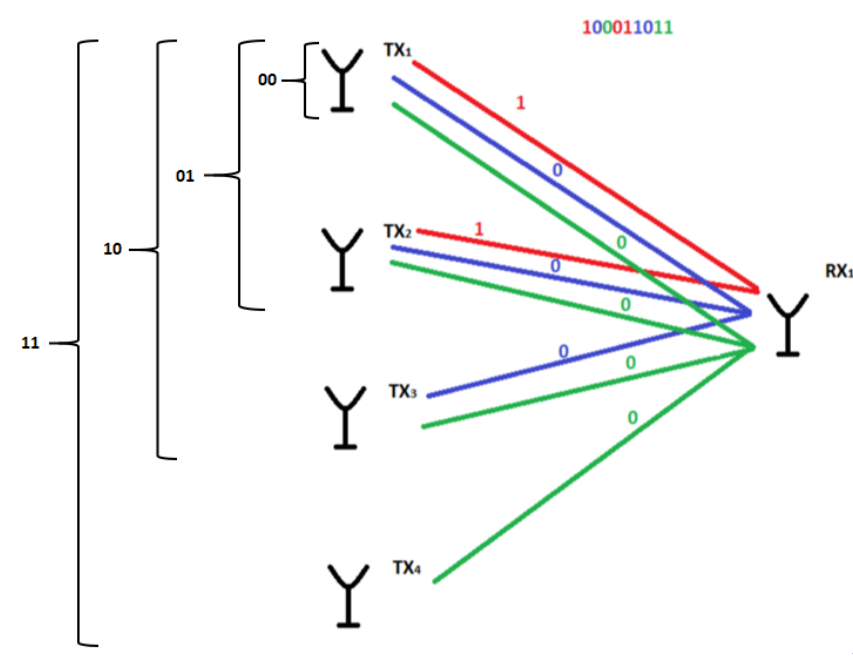

FIGURE 3. Simple visualization of the antenna activation process and mapping in MIMO-ANM scheme.

To illustrate the different models of possible received signals due to ANM mapping process, it is important to point out that for a different number of active antennas in usage, the corresponding received main signal expressions are different as listed in the following equations:

- When active antennas' number is one, the received signal is given as

$$
y_{1}=\sqrt{P} \times h_{11} \times x+w .
$$

- When active antennas' number is two, the received signal is given as

$$
y_{2}=\sqrt{\frac{P}{2}} \times\left(h_{11}+h_{12}\right) \times x+w .
$$

- When active antennas' number is three, the received signal is given as

$$
y_{3}=\sqrt{\frac{P}{3}} \times\left(h_{11}+h_{12}+h_{13}\right) \times x+w .
$$

- When active antennas' number is four, the received signal is given as

$$
y_{4}=\sqrt{\frac{P}{4}} \times\left(h_{11}+h_{12}+h_{13}+h_{14}\right) \times x+w .
$$

\section{B. THE DETECTION AT THE RECEIVER SIDE OF MIMO-ANM}

The corresponding receiver structure of the MIMO-ANM system is shown in Fig.4.

At the receiver side, the detection of the data bits conveyed by both antennas' number and conventional M-ary signal constellation symbols can be done jointly or individually (i.e., separately) in a successive or cascaded manner. The details of these two detection methods are given below.

To demodulate the data bits, we can perform joint, collective detection for both the BPSK symbols and ANM patterns 
TABLE 2. The calculated statistics of the effective channel amplitude of MISO-ANM with $T=4$

\begin{tabular}{|c|c|c|c|c|}
\hline Active Antennas Pattern (v) & $\left|h_{\text {eff }}\right|$ & Distribution & Scale $(\beta)$ & Mean Square $\left(\overline{\alpha^{2}}\right)$ \\
\hline$\left[\begin{array}{llll}1 & 0 & 0 & 0\end{array}\right]$ & $\left|h_{1}\right|$ & Rayleigh & 0.71 & 1 \\
\hline$\left[\begin{array}{llll}1 & 1 & 0 & 0\end{array}\right]$ & $\left|h_{1}+h_{2}\right|$ & Rayleigh & 1.0 & 2 \\
\hline$\left[\begin{array}{llll}1 & 1 & 1 & 0\end{array}\right]$ & $\left|h_{1}+h_{2}+h_{3}\right|$ & Rayleigh & 1.23 & 3 \\
\hline$\left[\begin{array}{llll}1 & 1 & 1 & 1\end{array}\right]$ & $\left|h_{1}+h_{2}+h_{3}+h_{4}\right|$ & Rayleigh & 1.42 & 4 \\
\hline
\end{tabular}

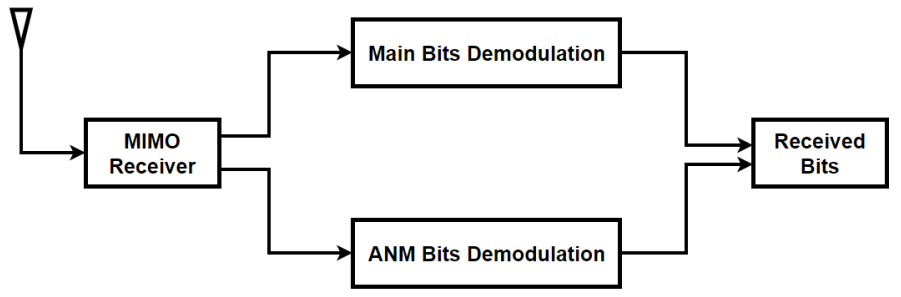

FIGURE 4. Receiver Structure of MIMO-ANM

all together in a combined manner, or use separate, sequential detection, where we first detect the active antennas' pattern, and after that perform BPSK detection followed by demodulation or de-mapping process to extract the transmitted data bits. Note that the complexity of joint detection is more than sequential detection because the number of for-loops required for joint (combined) detection is $T \times M$ compared to only $T+M$ for the case of cascaded (sequential) detection.

Without loss of generality, to decode the ANM Bits and Main Bits jointly, a Maximum Likelihood (ML) detector can be used to estimate and find out the active antennas' pattern and signal constellation point all together. The ML detector in this case is implemented according to the following formula.

$$
J_{v, x}=\min _{\hat{x}, \hat{v}}\left(\left\|y-\left(\sum_{i=1}^{\hat{v}} h_{1 i}\right) \hat{x}\right\|^{2}\right),
$$

where $v \in 1,2,3,4$ is the possible number of active transmit antennas, $y_{i} \in y_{1}, y_{2}, y_{3}, y_{4}$ is the received signal according to the possible antenna number usage, and $\hat{x_{i}}$ is the estimated BPSK symbol.

However, since the separate, sequential detection is much less complex due to requiring less number of for-loops than joint detection, it is more preferable to be used in practical scenarios that require low delay, less processing, and less power such as IoT devices. Accordingly, the ML detectors in the case of cascaded detection can be implemented according to the following formulas.

$$
\begin{aligned}
& J_{v}=\min _{\hat{v}}\left(\left\|y-\left(\sum_{i=1}^{\hat{v}} h_{1 i}\right) x\right\|^{2}\right), \\
& J_{x}=\min _{\hat{x}}\left(\left\|y-\left(\sum_{i=1}^{v} h_{1 i}\right) \hat{x}\right\|^{2}\right) .
\end{aligned}
$$

In the following, these formulas are extended according to active antenna patterns for a clearer presentation.
For the activation of one transmit antenna;

$$
\begin{aligned}
& J_{v=1}=\min _{\hat{v}}\left(\left\|y-\left(h_{11}\right) x\right\|^{2}\right) \\
& J_{x=1}=\min _{\hat{x}}\left(\left\|y-\left(h_{11}\right) \hat{x}\right\|^{2}\right)
\end{aligned}
$$

For the activation of two transmit antennas;

$$
J_{v=2}=\min _{\hat{v}}\left(\left\|y-\left(h_{11}+h_{12}\right) x\right\|^{2}\right)
$$

$$
J_{x=2}=\min _{\hat{x}}\left(\left\|y-\left(h_{11}+h_{12}\right) \hat{x}\right\|^{2}\right)
$$

For the activation of three transmit antennas;

$$
\begin{aligned}
& J_{v=3}=\min _{\hat{v}}\left(\left\|y-\left(h_{11}+h_{12}+h_{13}\right) x\right\|^{2}\right) \\
& J_{x=3}=\min _{\hat{x}}\left(\left\|y-\left(h_{11}+h_{12}+h_{13}\right) \hat{x}\right\|^{2}\right)
\end{aligned}
$$

For the activation of four transmit antennas;

$$
\begin{aligned}
& J_{v=4}=\min _{\hat{v}}\left(\left\|y-\left(h_{11}+h_{12}+h_{13}+h_{14}\right) x\right\|^{2}\right) \\
& J_{x=4}=\min _{\hat{x}}\left(\left\|y-\left(h_{11}+h_{12}+h_{13}+h_{14}\right) \hat{x}\right\|^{2}\right)
\end{aligned}
$$

The sequential detector is used in the simulation results section due to its many advantages, especially in reducing complexity compared to joint detectors.

\section{DIFFERENCES OF MIMO-ANM FROM VARIANTS OF MIMO-SM}

Although there are some schemes that might look similar to MIMO-ANM such as "Generalized Spatial Modulation" (GSM), "Variable Spatial Modulation" (VGSM), and "Extended Spatial Modulation" (ESM) [8]-[10], [12], MIMOANM still keeps its novelty. Each of the aforementioned schemes are explained below and the key differences between ANM and these schemes are highlighted in the following paragraphs.

In the GSM scheme, the system uses multiple antenna elements for each instance to simultaneously send the same data bits [17]. Within this feature, GSM may look quite similar to ANM. However, the key difference between ANM and GSM is that GSM uses a fixed number of active antennas given a certain number of available transmit antennas while ANM adjusts its number of active antennas according to the incoming bits and no information bits are sent by antennas' indices (just by their number instead). Table I in [17] shows that each group of bits is transmitted by a fixed combination 
of antennas which leads to a constraint in selecting the most convenient antennas for each case.

In another scheme that might seem similar to ANM called VGSM, the active transmit antennas are not fixed, meaning that the transmit antennas are activated according to the incoming bits and they may vary for each instance [18]. After the antenna activation, same data bits are sent from these activated antennas. The nature of VGSM looks even more similar to the ANM than GSM does. However, there is a vital difference between VGSM and ANM in addition to the difference between GSM and ANM. As clarified in Table 5.2 in [18], each group of active antennas is defined by the grouped bits with the indices of them. To be more clear, it can be said that even though the number of active antennas are not fixed, for each number of antenna usage cases, which antennas will be used is already defined, thus information bits are still sent by indices. However, in ANM, the antenna indices are kept undefined, only number of active antennas is used to convey additional bits. This provides ANM with flexibility to pick the particular antennas in the system that provide the highest channel quality.

Another scheme that can be confused with ANM concept is ESM. In this scheme, the transmit antennas are activated according to an incoming data stream in just the same way as VGSM. The fundamental point that distinguishes ESM from ANM is that, while the mapping numbers are assigned to each number of antennas in the ANM scheme, and no normalization is applied, in ESM, antennas' indices are still used to convey bits. Also, in ESM, the number of elements of the incoming data group is one, and the corresponding antennas in the system are activated without applying any mapping process. Besides, in this scheme, normalization is applied to the data vector when more than one element of the incoming data group is one [19]. A clearer view of ESM can be gained from Table I in [19].

\section{MIMO WITH ANTENNA NUMBER MODULATION AND} ADAPTIVE ANTENNA SELECTION (MIMO-ANM-AAS)

In this section, we introduce and explain the main advantageous property that MIMO-ANM has over MIMO-SM, which is the ability to make the selection of the active antennas to be not only data-dependent as is the case in MIMO$\mathrm{SM}$, but also simultaneously channel-dependent, which is not possible by MIMO-SM. This desirable property will result in enhancing the reliability performance of the system drastically due to exploiting spatial diversity, which was not possible with MIMO-SM, whose diversity gain is proven to be unity in many studies in the literature [8], [20], [21]. The resulting conceived adaptive transmission technique, in which the active transmit antennas are selected by both the ANM data bits and channel amplitude response, is named as MIMO with antenna number modulation and adaptive antenna selection (MIMO-ANM-AAS).

It should be pointed out that the proposed technique is different from the transmit antenna selection techniques conceived for MIMO-SM, such as [22]-[27], etc. in the sense that these techniques require the number of antennas at the transmitter to be greater than the number of antennas used for spatial modulation by which the index of an antenna (MIMO$\mathrm{SM}$ ) or indices of a group of antennas (MIMO-GSM) is/are made active at one transmission instance according to the incoming data bits.

The reason why MIMO-SM cannot be used with adaptive antenna selection (or transmit antenna selection) simultaneously is the fact that the selection of the active antennas' indices/positions in MIMO-SM is determined by the spatial data bits, whereas the selection of the active transmit antennas' indices/positions in transmit antenna selection techniques is determined by another metric such as Euclidean distance or the quality of the channel amplitude response. Therefore, the two selection processes are totally independent from each other, and thus cannot be used simultaneously together, leaving MIMO-SM with poor diversity gain.

The proposed MIMO-ANM-AAS technique works as follows:

1) By using the uplink channel in a Time division duplex (TDD) system, the receiver sends a known training sequence or reference sounding signal to allow the transmitter to estimate the channel amplitude responses of the antennas used in performing antenna number modulation.

2) From the above step, the transmitter will be able to obtain the channel amplitude values of the transmit antennas with respect to the receiver. For the case when the receiver is equipped with a single antenna and the transmitter with $T$ antennas, the channel amplitude vector is given by $|\mathbf{h}|=\left[\left|h_{11}\right|,\left|h_{12}\right|,\left|h_{13}\right|, \ldots,\left|h_{1 T}\right|\right] \in$ $\mathbb{C}^{1 \times T}$.

3) The transmitter sorts the channel amplitude values in the vector $|\mathbf{h}|$ in an ascending order (from largest to smallest).

4) To improve the reliability performance at the receiver, the transmitter tries to maximize the SNR of the receiver, which automatically results in reducing the BER and thus improving reliability. Since the SNR is function of the channel power gains values, the transmitter will consider the strategy ${ }^{3}$ of selecting the antennas corresponding to the largest channel gains, and use them for number modulation. This is possible because number modulation does not care about the indices of the antennas, but rather on the number only.

5) More precisely, in MIMO-ANM-AAS, after the decision of selecting the number of antennas that will be used for data transmission by ANM bits, the decision of specifying which antennas' positions/indices will be used for transmission is exclusively decided by the channel coefficients quality of transmit antenna

\footnotetext{
${ }^{3}$ It is worth mentioning that the strategy of selecting the transmit antennas according to the largest channel gains is much simpler and has lower cost and complexity compared to using other strategies such as Euclidean distancebased or capacity-based transmit antenna selection schemes, which were used in the literature with MIMO-SM [22]-[27].
} 
elements.

The resulting problem of the optimal selection of antenna positions corresponds to solving the below optimization equation for all possible antenna combinations:

$$
\left\{v_{1}^{o p t}, \cdots, v_{V}^{o p t}\right\}=\arg \max _{\left\{v_{1}, \cdots, v_{V}\right\} \in \mathcal{A}_{V}} S N R_{\left[v_{1}, \cdots, v_{V}\right]},
$$

where $\mathcal{A}_{V}$ represents the set of all possible active antenna patterns' combinations, and $S N R_{\left[v_{1}, \cdots, v_{V}\right]}$ is the sum of SNRs of the $V$ selected antennas in each transmission block.

Now, since uniform power allocation is used for all active antennas, the aforementioned problem boils down to selecting the antennas corresponding to the best subchannel gains. This can be given as below

$$
\left\{v_{1}^{o p t}, \cdots, v_{V}^{o p t}\right\}=\arg \max _{\left\{v_{1}, \cdots, v_{V}\right\} \in \mathcal{A}_{V}}\left|h_{1 V}\right|_{\left[v_{1}, \cdots, v_{V}\right]},
$$

Example: In a MIMO-ANM-AAS system that consists of four transmit antennas and one receive antenna, and ANM bits group to be transmitted is 00 , then only one antenna will be activated, but in this case there are four different combinations of which antenna position can be activated. These antenna combinations can be specified as $\left[\begin{array}{llll}1 & 0 & 0 & 0\end{array}\right]$ which means that the channel quality of the first transmit antenna is the highest one among four transmit antennas. Dependently, transmission of "main bits" symbol is operated from the first antenna. On the other hand, when the pattern $\left[\begin{array}{llll}0 & 1 & 0 & 0\end{array}\right]$ is selected, this means that the channel quality of second transmit antenna is the highest one among four transmit antennas. Dependently, transmission of "main bits" symbol is operated from second antenna. Also, when [0 $\left.\begin{array}{lll}0 & 1 & 0\end{array}\right]$ is chosen which means that the channel quality of third transmit antenna is the highest one among four transmit antennas transmission of "main bits" symbol is operated from third antenna, and lastly when [0 000 1] is selected, which means that the channel quality of fourth transmit antenna is the highest one among four transmit antennas, transmission of "main bits" symbol is operated from fourth antenna.

6) The possible, different active antenna patterns corresponding simultaneously to both of the largest channel gains and modulated data bits by antennas' number are shown in Table II. The set of active antenna patterns may be computed at the transmitter for every coherence interval and the receiver just needs to estimate the number of active antennas in the received signal, and then de-map it to the ANM bits. After that, the receiver estimates the BPSK data symbol and de-map it to the main data bits. As an example, if the incoming data bits modulated by antennas' number is 00 , the transmitter will activate only one antenna that corresponds to the location of the largest channel gain among all the $T$ antennas' channel amplitude responses.

TABLE 3. MIMO-ANM-AAS mapper with $N_{2}=2$ bits $\& T=4$ antennas, where the final active antenna pattern is determined by the largest channel gains

\begin{tabular}{|c|c|}
\hline ANM bits $\left(N_{2}\right)$ & Possible Active Antennas Pattern (v) \\
\hline$\left[\begin{array}{ll}0 & 0\end{array}\right]$ & {$\left[\begin{array}{llll}1 & 0 & 0 & 0\end{array}\right]$} \\
\hline$\left[\begin{array}{ll}0 & 0\end{array}\right]$ & {$\left[\begin{array}{llll}0 & 1 & 0 & 0\end{array}\right]$} \\
\hline$\left[\begin{array}{ll}0 & 0\end{array}\right]$ & {$\left[\begin{array}{llll}0 & 0 & 1 & 0\end{array}\right]$} \\
\hline$\left[\begin{array}{ll}0 & 0\end{array}\right]$ & {$\left[\begin{array}{llll}0 & 0 & 0 & 1\end{array}\right]$} \\
\hline$\left[\begin{array}{ll}0 & 1\end{array}\right]$ & {$\left[\begin{array}{llll}1 & 1 & 0 & 0\end{array}\right]$} \\
\hline$\left[\begin{array}{ll}0 & 1\end{array}\right]$ & {$\left[\begin{array}{llll}1 & 0 & 1 & 0\end{array}\right]$} \\
\hline$\left[\begin{array}{ll}0 & 1\end{array}\right]$ & {$\left[\begin{array}{llll}1 & 0 & 0 & 1\end{array}\right]$} \\
\hline$\left[\begin{array}{ll}0 & 1\end{array}\right]$ & {$\left[\begin{array}{llll}0 & 0 & 1 & 1\end{array}\right]$} \\
\hline$\left[\begin{array}{ll}0 & 1\end{array}\right]$ & {$\left[\begin{array}{llll}0 & 1 & 0 & 1\end{array}\right]$} \\
\hline$\left[\begin{array}{ll}0 & 1\end{array}\right]$ & {$\left[\begin{array}{llll}0 & 1 & 1 & 0\end{array}\right]$} \\
\hline$\left[\begin{array}{ll}1 & 0\end{array}\right]$ & {$\left[\begin{array}{llll}1 & 1 & 1 & 0\end{array}\right]$} \\
\hline$\left[\begin{array}{ll}1 & 0\end{array}\right]$ & {$\left[\begin{array}{llll}1 & 1 & 0 & 1\end{array}\right]$} \\
\hline [1 0 l & {$\left[\begin{array}{llll}1 & 0 & 1 & 1\end{array}\right]$} \\
\hline [1 0 ] & {$\left[\begin{array}{llll}0 & 1 & 1 & 1\end{array}\right]$} \\
\hline$\left[\begin{array}{ll}1 & 1\end{array}\right]$ & {$\left[\begin{array}{llll}1 & 1 & 1 & 1\end{array}\right]$} \\
\hline
\end{tabular}
among all possible antenna numbers

\section{PERFORMANCE ANALYSIS OF MIMO-ANM}

\section{A. STATISTICS OF THE EFFECTIVE INSTANTANEOUS SNR}

The proposed MIMO-ANM modulation scheme causes some changes to the original fading distribution's parameters, where the observed effective fading parameters at the receiver differ for different antenna number activation patterns. This is due to the fact that at each transmission slot (or channel use), different number of channels are used to transmit data symbols according to the incoming ANM bits. Thus, the fading distribution parameters are expected to change as the antenna number activation pattern changes.

In order to obtain the distributions of the amplitude and power of the faded channels, we use numerical data fitting methods. Particularly, the distributions are obtained by simulating 320000 realizations generated from a standard Rayleigh fading distribution that is obtained from taking the amplitude of a circularly symmetric Gaussian distribution with zero mean and unity variance. Then, the proposed MIMO-ANM scheme is applied for selecting the antennas' number to be used for data transmission based on the incoming ANM bits. Last, fitting tools are utilized to obtain the best matching distribution's parameters for the fading amplitude and power of the actual, effective used channels.

Since selecting the transmit antennas number pattern is determined by ANM bits, thus this will correspond to a random channels set with respect to the receiver. Therefore, the distribution is intuitively anticipated to be similar to the original assumed fading distribution, i.e., Rayleigh, but with different parameters (i.e., scale factors and means). Our obtained results confirm this intuition and demonstrate that the effective distribution of the channel amplitude is approximately Rayleigh distributed (same as the original one) with different scale factors and means as shown in Table II. Also, 
the effective distribution of the channel power is exponential with mean factor $\psi$, which is equal to the mean square of the effective channel amplitude $\left(\psi=\bar{\alpha}^{2}\right)$ presented in the last column of Table II. Mathematically, the amplitude channel distribution can be given as

$$
f_{\alpha}(\alpha)=\frac{\alpha}{\beta^{2}} \exp \left(-\frac{\alpha^{2}}{2 \beta^{2}}\right),
$$

where $\beta$ is the scale parameter and $\alpha=\left|h_{e f f}\right|$ is the instantaneous amplitude of the effective obtained channel distribution, which is Rayleigh in this case. Also, $\Omega=\bar{\alpha}^{2}$ is the mean square variable of $\alpha$, which is also equal to $\psi$ obtained by fitting the effective channel power. For the possible antenna activation patterns resulting from the proposed MIMO-ANM, we have the distribution parameters given in Table II.

The PDF of $\gamma=\frac{\left|H_{e f f}\right|^{2} P}{\sigma^{2}}$ can be determined by using a change of variables in the expression for the fading distribution $f_{\alpha}(\alpha)$ of $\alpha$, yielding

$$
f_{\gamma}(\gamma)=\frac{f_{\alpha}\left(\sqrt{\frac{\Omega \gamma}{\bar{\gamma}}}\right)}{\frac{1}{\beta^{2}} \sqrt{\frac{\bar{\gamma} \gamma}{\Omega}}}
$$

By considering the case of any antennas' activation pattern in MIMO-ANM scheme, the approximated PDF of the effective SNR, $f_{\gamma}(\gamma)$, can be given in an exponential distribution form. For the special case, when only one antenna is active, which corresponds to $\beta=0.71$, the instantaneous SNR distribution formula can be found as

$$
f_{\gamma}(\gamma)=\left(\frac{1}{\Omega \bar{\gamma}}\right) \exp \left(\frac{\gamma}{\Omega \bar{\gamma}}\right) .
$$

Using the above calculated distribution functions, we can now evaluate and analyze the BER performance of the proposed MIMO-ANM conveniently, enabling us to examine the advantages of the proposed scheme.

\section{B. ERROR PERFORMANCE ANALYSIS OF MIMO-ANM}

Computation of the analytical performance of MIMO-ANM is not straightforward. This is so because there are two estimation processes that are involved: 1) estimation of the number of transmit antennas, and 2) estimation of the transmitted symbol. The two processes are assumed to be independent in the calculation. However, this is not generally correct. For instance, if the channel paths are correlated, the two estimation processes will be dependent, and the following derived equation is the upper bound on the true performance in such channel conditions.

The transmitted bits are correctly recovered only if both estimates are correct. To compute the overall probability of error, let $A 1$ and $A 2$ represent the first and the second estimation processes, respectively. Since the first block of bit-substream, which is denoted by $B_{A N M}$, and the second block of bit-substream, which is denoted by $B_{B P S K}$, are not equal in bits sequence length, their respective probabilities are $P(A 1)=2 / 3$ and $P(A 2)=1 / 3$. Now, let $P_{A N M}(E)$ be the error probability for $A 1$ (i.e., the antenna pattern activity) and $P_{B P S K}(E)$ be the error probability for $A 2$ (i.e., the BPSK symbol recovery) at the receiver side. The overall error probability $P_{t o t}(E)$ can be formulated using the law of total probability as follows:

$$
\begin{gathered}
P_{t o t}(E)=P_{t o t}\left(E \mid A_{1}\right) P\left(A_{1}\right)+P_{t o t}\left(E \mid A_{2}\right) P\left(A_{2}\right) \\
P_{t o t}(E)=\frac{2}{3} P_{A N M}(E)+\frac{1}{2} P_{B P S K}(E)
\end{gathered}
$$

In what follows, the error probability of each estimation process is considered separately using both power reallocation policy (PRP) and power saving policy (PSP).

\section{ANALYTICAL SER OF THE TRANSMITTED SYMBOL ESTIMATION PROCESS}

The estimation of the transmitted symbol for any M-QAM is a $1 \times T_{n}$ detection in a MISO-ANM system, where $T_{n}$ represents the number of active antennas at the transmitter for a certain channel use. This is so because only the corresponding element in the resulting vector is considered for the estimation process.

The closed-form SER expression for M-ary QAM over a Rayleigh fading channel is given as [28]

$$
\begin{aligned}
P_{s} & =2\left(\frac{\sqrt{M}-1}{\sqrt{M}}\right)\left(1-\sqrt{\frac{1.5 \bar{\gamma}_{s}}{M-1+1.5 \bar{\gamma}_{s}}}\right)-\left(\frac{\sqrt{M}-1}{\sqrt{M}}\right)^{2} \\
& \times\left[1-\sqrt{\frac{1.5 \bar{\gamma}_{s}}{M-1+1.5 \bar{\gamma}_{s}}}\left(\frac{4}{\pi} \tan ^{-1} \sqrt{\frac{M-1+1.5 \bar{\gamma}_{s}}{1.5 \bar{\gamma}_{s}}}\right)\right]
\end{aligned}
$$

Note that $\bar{\gamma}_{s}=\bar{\gamma} \log _{2}(M)$, where $\bar{\gamma}_{s}$ denotes the average SNR per symbol. Also, note that for uncoded data the average BER can be approximated as $P_{b}=\frac{P_{s}}{\log _{2} M}$, which becomes $P_{b}=\frac{P_{s}}{2}$ for $M=4$ and $P_{b}=P_{s}$ for $M=2$.

Hence, $P_{q}$ for 4-QAM can be written as follows

$$
P_{q}=-\frac{1}{8}-\frac{1}{2} \sqrt{\frac{\bar{\gamma}_{s}}{2+\bar{\gamma}_{s}}}+\frac{1}{2 \pi} \sqrt{\frac{\bar{\gamma}_{s}}{2+\bar{\gamma}_{s}}} \tan ^{-1} \sqrt{\frac{2+\bar{\gamma}_{s}}{\bar{\gamma}_{s}}},
$$

whereas, $P_{q}$ for BPSK can be written as follows

$$
P_{q}=P_{B P S K}(E)=\frac{1}{2}\left(1-\sqrt{\frac{\bar{\gamma}_{s}}{1+\bar{\gamma}_{s}}}\right) .
$$

\section{ANALYTICAL ERROR OF THE NUMBER OF ACTIVE TRANSMIT ANTENNA ESTIMATION PROCESS}

In the following, the computation of $P_{A N M}(E)$ is considered. For simplicity, only four transmit antennas are considered for the derivation, and the result is later generalized to any number of transmit antennas.

To illustrate, assume that a sequence of data bits is mapped to symbol $s_{2}$ from a square QAM or BPSK constellation, and then to the second transmit antenna activation pattern (i.e., 
[1100]). Then, the received vector $y=\left(h_{1}+h_{2}\right) x_{2}+n$ appears at the input of the receive antennas.

The estimation process of the antennas number pattern activity using coherent detector is similar to the detection of multiple amplitude shift keying (M-ASK) modulation [29]. For the case of having four possible active antenna number patterns, the conditional pair-wise error ratio expression, which is somewhat similar to the conditional SER over a Rayleigh fading channel, can be written as follows

$$
P_{e / \gamma_{s}}=2 \frac{(T-1)}{T} Q_{f}\left(\sqrt{2 G \times \gamma_{s}}\right)
$$

where $G=3 /\left(T^{2}-1\right)$ and $Q_{f}(x)$ is the $Q$ function defined as

$$
Q_{f}(x)=\frac{1}{\sqrt{2 \pi}} \int_{x}^{\infty} e^{-\frac{u^{2}}{2}} d u .
$$

Using the alternative representation of the Gaussian function, we obtain the conditional SER in the desired product form as

$$
P_{e / \gamma_{s}}=2 \frac{(T-1)}{T \pi} \int_{0}^{\frac{\pi}{2}} \exp \left(\frac{-G \overline{\gamma_{s}}}{\sin ^{2}(\phi)}\right) d \phi
$$

Now, the unconditional error probability can be obtained as

$$
\begin{gathered}
P_{e}=\int_{0}^{\infty} P_{e / \gamma_{s}} p_{\gamma}\left(\gamma_{s} ; \beta, \alpha\right) d \gamma_{s} \\
P_{e}=\int_{0}^{\infty} 2 \frac{(T-1)}{T} Q_{f}\left(\sqrt{2 G \times \gamma_{s}}\right) p_{\gamma}\left(\gamma_{s} ; \beta, \alpha\right) d \gamma_{s} \\
P_{e}=P_{A N M}(E)=\frac{T-1}{T}\left(1-\sqrt{\frac{G \bar{\gamma}_{s}}{1+G \bar{\gamma}_{s}}}\right)
\end{gathered}
$$

where $\bar{\gamma}_{s}=\frac{\overline{\alpha^{2}}}{2 \beta^{2}} \frac{\bar{E}_{s}}{N_{s}^{s}}$ denotes the average SNR per symbol, and $\alpha$ and $E_{s}$ are the fading amplitude and the average symbol energy, respectively.

By substituting (26), and (32) into (23), the overall error probability using both power reallocation policy (PRP) and power saving policy (PSP) can be easily formulated.

\section{AVERAGE BER PERFORMANCE OF ALL POSSIBLE ANTENNA NUMBER ACTIVATION PATTERNS}

In MIMO-ANM, there are two possible reasons for error. One of them is the error that occurs during the detection of the main bits, and the other is the error that occurs during the detection of the ANM bits.

The theoretical bit error rate (BER) arising from the detection of the ANM bits for the cases of different number of antennas can be obtained from (24) with the note that each detection error in the actual active pattern results in one bit error if the wrongly detected pattern is adjacent to the actual transmitted pattern, and in two bits' errors if the wrongly detected pattern is not adjacent. This is due to having gray like mapping between ANM bits and the active patterns as shown in Table I. These BER formulas of ANM bits are derived and given by the following expressions:

For the case of activating one transmit antenna:

$$
B E R_{A N M-1}=\frac{3}{2 \times 4} \times\left(1-\sqrt{\frac{\frac{\frac{3}{15} \times E_{b}}{2 \times 0.71^{2} \times N_{0}}}{\frac{3}{15} \times E_{b}}}\right)
$$

For the case of two transmit antenna:

$$
B E R_{A N M-2}=\frac{3}{2 \times 4} \times\left(1-\sqrt{\frac{\frac{\frac{3}{15} \times 2 \times E_{b}}{2 \times 1.0^{2} \times N_{0}}}{\frac{3}{15 \times 2 \times E_{b}}}}\right)
$$

For the case of three transmit antenna:

$$
B E R_{A N M-3}=\frac{3}{2 \times 4} \times\left(1-\sqrt{\frac{\frac{\frac{3}{15} \times 3 \times E_{b}}{2 \times 1.23^{2} \times N_{0}}}{\frac{\frac{3}{15} \times 3 \times E_{b}}{2 \times 1.23^{2} \times N_{0}}+1}}\right)
$$

For the case of four transmit antenna:

$$
B E R_{A N M-4}=\frac{3}{2 \times 4} \times\left(1-\sqrt{\frac{\frac{\frac{3}{15} \times 4 \times E_{b}}{2 \times 1.42^{2} \times N_{0}}}{\frac{3}{15} \times 4 \times E_{b}}}\right)
$$

After the BER due to each case is calculated separately, the average value of these results is taken as the BER of the ANM Bits as given below.

$$
B E R_{A N M}=\frac{\sum_{i=1}^{4} B E R_{A N M-i}}{4} .
$$

As for the main bits group, since the Main bits part is also dependent on the number of antennas as the mean $(\psi)$ of the channel power distribution changes with the number of active transmit antennas, coefficient $\psi$ is used in the derivation to reflect the effect of the channel on the BER.

The theoretical BER formulas of Main bits for different number of active transmit antennas can be obtained from (18). The BER formulas of Main bits for different transmit antenna number activation patterns are calculated and given as follows:

For the case of one transmit antenna $(\psi=1)$ :

$$
B E R_{\text {Main }-1}=\frac{1}{2} \times\left(1-\sqrt{\frac{\frac{E_{b}}{2 \times 0.71^{2} \times N_{0}}}{\frac{E_{b}}{2 \times 0.71^{2} \times N_{0}}+1}}\right) .
$$

For the case of two transmit antennas $(\psi=2)$ :

$$
B E R_{\text {Main }-2}=\frac{1}{2} \times\left(1-\sqrt{\frac{\frac{2 \times E_{b}}{2 \times 1.0^{2} \times N_{0}}}{\frac{2 \times E_{b}}{2 \times 1.0^{2} \times N_{0}}+1}}\right) .
$$

For the case of three transmit antennas $(\psi=3)$ :

$$
B E R_{\text {Main }-3}=\frac{1}{2} \times\left(1-\sqrt{\frac{\frac{3 \times E_{b}}{2 \times 1.23^{2} \times N_{0}}}{\frac{3 \times E_{b}}{2 \times 1.23^{2} \times N_{0}}+1}}\right) .
$$


For the case of four transmit antennas $(\psi=4)$ :

$$
B E R_{\text {Main }-4}=\frac{1}{2} \times\left(1-\sqrt{\frac{\frac{4 \times E_{b}}{2 \times 1.42^{2} \times N_{0}}}{\frac{4 \times E_{b}}{2 \times 1.42^{2} \times N_{0}}+1}}\right) .
$$

After the BER due to each case is calculated separately ${ }^{4}$ the average value of these results is taken as the BER of the Main bits as given below.

$$
B E R_{\text {Main }}=\frac{\sum_{i=1}^{4} B E R_{\text {Main }-i}}{4} .
$$

After the BERs of the Main bits group and the ANM bits group have been calculated, the average of these values is calculated to find the theoretical total average BER of the MIMO-ANM system.

$$
B E R_{M I M O-A N M}=\frac{2}{3} \times B E R_{A N M}+\frac{1}{3} \times B E R_{M a i n} .
$$

Note that (35) is applicable to the case when BPSK modulation is employed and four transmit antennas are used by number modulation. For instance, when QPSK is used, the number of bits transmitted by signal constellations will be exactly equal to the number of bits transmitted by number modulation, and thus the above BER would change to become as follows

$$
B E R_{M I M O-A N M}=\frac{1}{2} \times B E R_{A N M}+\frac{1}{2} \times B E R_{M a i n} \text {. }
$$

\section{PERFORMANCE DEMONSTRATION}

It is worth mentioning that the spectral efficiency and power efficiency of the proposed MIMO-ANM scheme is obviously the same as that provided by conventional MIMO-SM scheme. Therefore, the focus of the performance demonstration and comparison will be on the reliability aspect. Accordingly, in this section, the performance results in terms of BER are obtained by conducting Monte-Carlo simulations for the two proposed modulation schemes MIMO-ANM and MIMO-ANM-AAS over a wireless Rayleigh fading channel. In the first part of the simulation setup, the number of transmit antennas used for number modulation is considered to be four $(T=4)$, whereas the number of receive antenna is assumed to be one $(R=1)$.

This purposefully selected setup is considered as so in order to concentrate the attention on the fundamental concepts of the proposed schemes, and to simplify the comparison with the other competitive schemes available in the literature,

\footnotetext{
${ }^{4}$ It should be mentioned that the derived formulas given in (30), (31), (32), and (33) are found to give identical, equal BER performances, although they have different parameters. This is due to the fact that transmitting the same information symbol from different transmit antennas without beamforming or space-time coding does not improve the performance whatsoever as the channels are added randomly to each other. Thus, the effective channel in a two, or three, or four transmit antennas is again a Rayleigh distributed channel but with different parameters (i.e., $\beta \& \overline{\alpha^{2}}$ ). Therefore, the BER performance is identical to the case of SISO transmission over a Rayleigh fading channel, given that the detection of the number of active antennas is perfectly correct, which can happen at high SNR values.
}

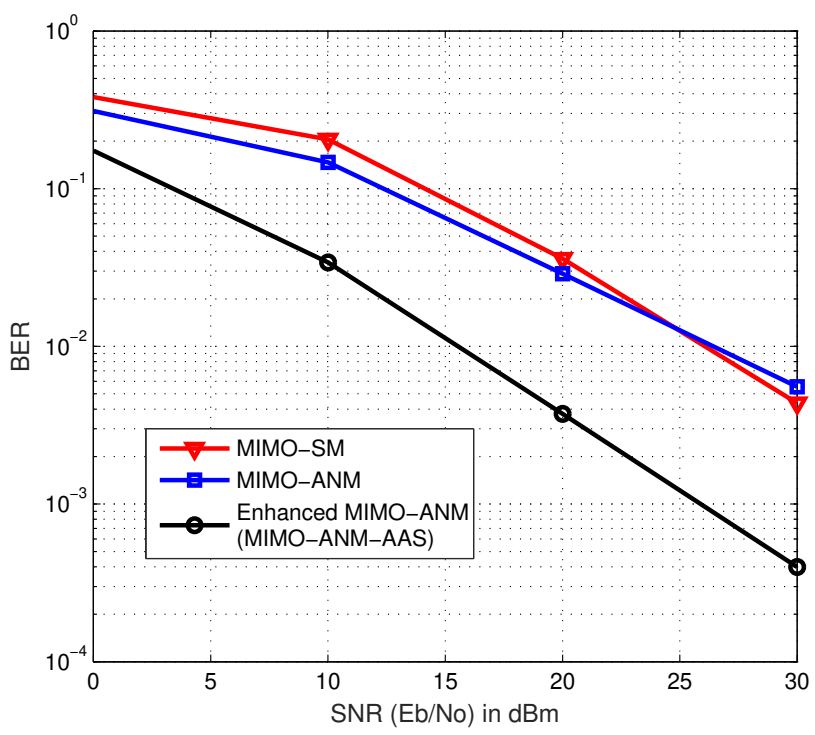

FIGURE 5. BER of MIMO-ANM vs. SNR (Eb/N0) compared to that of MIMO-ANM-AAS (Enhanced MIMO-ANM) and MIMO-SM under power saving policy (PSP).

such as MIMO-SM. The parameters used in the simulations are shown and summarized in Table 4.

TABLE 4. Simulation Parameters

\begin{tabular}{|c|c|}
\hline Modulation Type & BPSK $(M=2)$ \\
\hline Number of Symbols & $10^{6}$ per iteration \\
\hline Number of Transmit Antennas & 4 \\
\hline Number of Receive Antennas & 1 \\
\hline Number of available antennas for ANM & 4 \\
\hline Number of ANM mapping bits & 2 \\
\hline Wireless channel & Block Rayleigh fading \\
\hline
\end{tabular}

The numerically-simulated BER performance curves of the proposed MIMO-ANM and MIMO-ANM-AAS schemes are shown in Fig. 5. Besides, the figure shows a comparison of the proposed schemes with the conventional MIMOSM scheme, where MIMO-ANM-AAS (Enhanced MIMOANM) showed superior and better BER performance. Particularly, at a $\mathrm{BER}=10^{-2}$, MIMO-ANM-AAS scheme is shown to be able to achieve a gain of more than $10 \mathrm{dBs}$ compared to conventional MIMO-SM. This is very significant gain that can extend the coverage of the system or save the transmit power by orders of magnitude while satisfying and meeting the requirements of a target application or service.

The average error rates associated with the Main Bits, which are modulated by BPSK, and ANM Bits, which are modulated by antenna number, in MIMO-ANM-AAS are presented in Fig. 6. As the SNR value is varied from 0 to 30, the BERs of the Main bits and the ANM bits significantly fall rapidly as compared with conventional MIMO-SM.

As depicted, even though in a four transmit antenna system with BPSK modulation both MIMO-SM and MIMO-ANM 


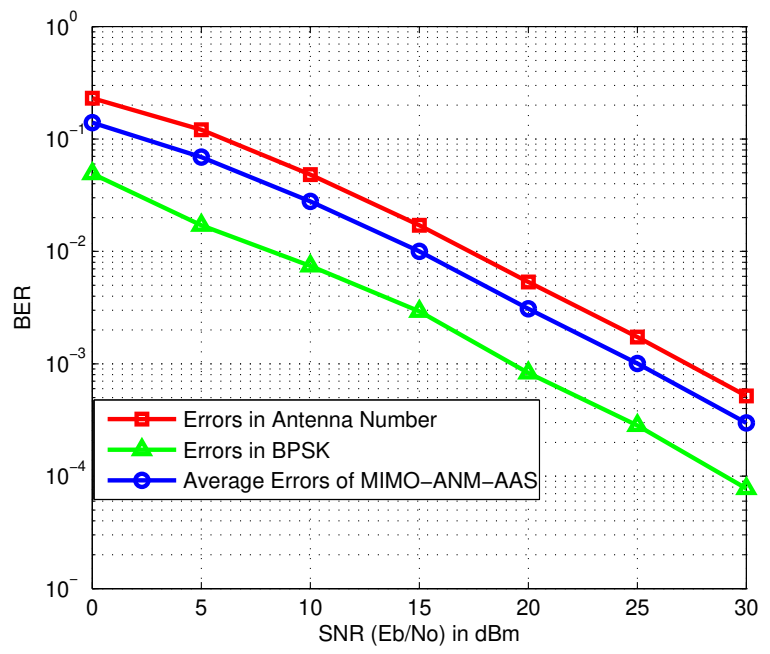

FIGURE 6. BER of MIMO-ANM-AAS (Enhanced MIMO-ANM) vs. SNR (Eb/NO) under power saving policy (PSP).

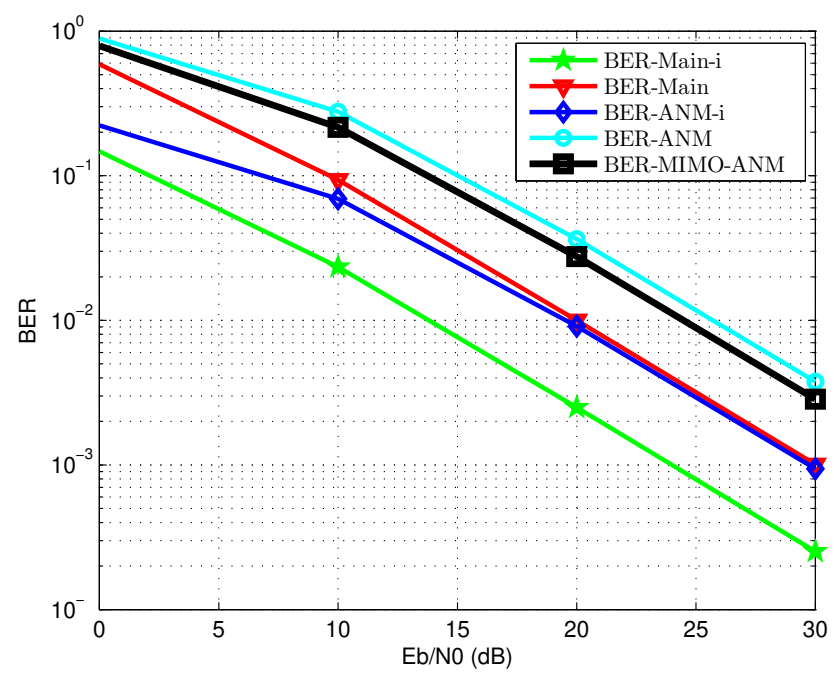

FIGURE 7. Theoretically derived BER formulas of MIMO-ANM vs. SNR $(\mathrm{Eb} / \mathrm{NO}$ ) without power reallocation under power saving policy (PSP).

offer to convey three bits at a time, while having also a similar BER performance, MIMO-ANM is also able to decrease the transceiver complexity similar to MIMO-SM. This is so because MIMO-ANM has only one radio frequency (RF) chain that is connected with multiple antenna using RF switches. On the other hand, MIMO-ANM-AAS offers a significantly higher reliability than that offered by MIMOSM because of its utilization of diversity, where it allows and enables the selection of the antennas corresponding to the best channel gains to send data information symbols, resulting in further improvement in the BER performance due to delivering coding/diversity gains as demonstrated in Fig. 5

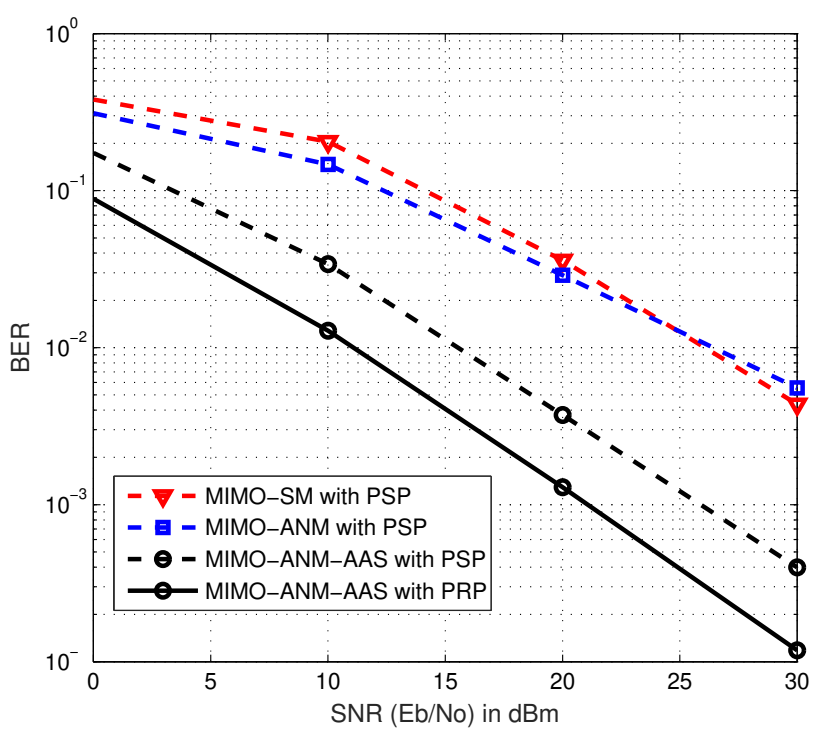

FIGURE 8. BER performance of MIMO-ANM-AAS (Enhanced MIMO-ANM) under power reallocation policy (PRP) compared to that under power saving policy (PSP).

and Fig. 6.

Fig. 7 exhibits the BER performance curves of the theoretically derived BER formulas. The cyan color curve (BERANM) represents the theoretical BER of ANM Bits, red color curve (BER-Main) represents the theoretical BER of Main (BPSK) bits, and black bold color curve (BER-MIMOANM) represents the overall average theoretical BER of MIMO-ANM under power saving policy, which shows similar behavior to the numerically simulated BER performance that is shown in Fig. 5. On the other hand, the blue color curve (BER-ANM-i) represents the theoretical BER of ANM Bits, and green color curve (BER-Main-i) represents the theoretical BER of Main (BPSK) bits under power reallocation policy.

Fig. 8 shows the significant improvement in the BER performance of MIMO-ANM-AAS when power reallocation policy is considered, where the saved power due to transmitting two additional bits (ANM bits) by only changing the number of antennas without the need to transmit additional power is reallocated to the transmitted BPSK data symbol. Consequently, the linear power allocated to a single BPSK symbol will be trebled, which will result in enhancing the BER by more than $4 \mathrm{~dB}$ as compared to the case of PSP as can be seen from Fig. 8 .

\section{CONCLUSION}

In this paper, a novel data transmission method called MIMO-ANM has been introduced. The underlying principle of MIMO-ANM is to utilize number of antennas as another degree of freedom that can be used for conveying extra data bits besides those transmitted by conventional M-ary 
PSK/QAM symbols. The key advantage of this proposed system is that MIMO-ANM does not dictate which antennas will be used for data transmission, but rather dictates the number of antennas to be used for transmission. On the contrary to the GSM, VGSM, ESM, this characteristic of MIMO-ANM enables the selection of the best antennas in the system that provide the highest signal quality during transmission.

\section{ACKNOWLEDGMENT}

We would like to thank Seyit Karatepe and Ali Yildiz for their efforts in reviewing and checking the Matlab codes used for simulating the proposed MIMO-ANM-AAS.

\section{REFERENCES}

[1] Jehad M. Hamamreh, Abdulwahab Hajar, and Mohamedou Abewa. Orthogonal frequency division multiplexing with subcarrier power modulation for doubling the spectral efficiency of $6 \mathrm{G}$ and beyond networks. Transactions on Emerging Telecommunications Technologies, 31(4):e3921, 2020.

[2] Abdulwahab Hajar, Jehad M Hamamreh, Mohamedou Abewa, and Youcef Belallou. A spectrally efficient OFDM-based modulation scheme for future wireless systems. In 2019 Scientific Meeting on Electrical-Electronics \& Biomedical Engineering and Computer Science (EBBT), pages 1-4. IEEE, 2019.

[3] S. Yang and L. Hanzo. Fifty years of MIMO detection: The road to largescale mimos. IEEE Communications Surveys Tutorials, 17(4):1941-1988, Fourthquarter 2015.

[4] Arogyaswami J Paulraj and Thomas Kailath. Increasing capacity in wireless broadcast systems using distributed transmission/directional reception (DTDR), September 6 1994. US Patent 5,345,599.

[5] Andreas F Molisch and Moe Z Win. MIMO systems with antenna selection-an overview. IEEE microwave magazine, 5(1):46-56, 2004.

[6] Ertuğrul Başar. Multiple-input multiple-output ofdm with index modulation. IEEE Signal Processing Letters, 22(12):2259-2263, 2015.

[7] Harald Haas, S Sinanovic, CW Ahn, and S Yun. Spatial modulation. IEEE Trans. Veh. Technol., 57(4):2228-2241, 2008.

[8] M. Wen, B. Zheng, K. J. Kim, M. Di Renzo, T. A. Tsiftsis, K. Chen, and N. Al-Dhahir. A survey on spatial modulation in emerging wireless systems: Research progresses and applications. IEEE Journal on Selected Areas in Communications, 37(9):1949-1972, Sep. 2019.

[9] N. Ishikawa, S. Sugiura, and L. Hanzo. 50 years of permutation, spatial and index modulation: From classic RF to visible light communications and data storage. IEEE Communications Surveys Tutorials, 20(3):19051938, thirdquarter 2018.

[10] Ertugrul Basar, Miaowen Wen, Raed Mesleh, Marco Di Renzo, Yue Xiao, and Harald Haas. Index modulation techniques for next-generation wireless networks. IEEE Access, 5:16693-16746, 2017.

[11] Ahmad M Jaradat, Jehad M Hamamreh, and Hüseyin Arslan. Modulation options for ofdm-based waveforms: Classification, comparison, and future directions. IEEE Access, 7:17263-17278, 2019.

[12] Space Modulation Transmission and Reception Techniques, chapter 3 , pages 35-83. John Wiley Sons, Ltd, 2018

[13] Ali Ghrayeb. A survey on antenna selection for mimo communication systems. In 2006 2nd International Conference on Information \& Communication Technologies, volume 2, pages 2104-2109. IEEE, 2006.

[14] Beng Soon Tan, Kwok Hung Li, and Kah Chan Teh. Transmit antenna selection systems: A performance comparison of different types of receiver schemes. IEEE Vehicular Technology Magazine, 8(3):104-112, 2013.

[15] Ahmad M Jaradat, Jehad M Hamamreh, and Huseyin Arslan. OFDM with subcarrier number modulation. IEEE Wireless Communications Letters, 7(6):914-917, 2018.

[16] A. M. Jaradat, J. M. Hamamreh, and H. Arslan. OFDM with hybrid number and index modulation. IEEE Access, 8:55042-55053, 2020.

[17] Abdelhamid Younis, Nikola Serafimovski, Raed Mesleh, and Harald Haas. Generalised spatial modulation. In 2010 Conference Record of the Forty Fourth Asilomar Conference on Signals, Systems and Computers, pages 1498-1502. IEEE, 2010.

[18] Abdelhamid Younis. Spatial modulation: Theory to practice. 2014.
[19] JM Luna-Rivera and MG Gonzalez-Perez. An improved spatial modulation scheme for mimo channels. In 2012 6th European Conference on Antennas and Propagation (EUCAP), pages 1-5. IEEE, 2012.

[20] T. Handte, A. Muller, and J. Speidel. Ber analysis and optimization of generalized spatial modulation in correlated fading channels. In 2009 IEEE 70th Vehicular Technology Conference Fall, pages 1-5, Sep. 2009.

[21] M. Di Renzo and H. Haas. On transmit diversity for spatial modulation mimo: Impact of spatial constellation diagram and shaping filters at the transmitter. IEEE Transactions on Vehicular Technology, 62(6):25072531, July 2013.

[22] P. Yang, Y. Xiao, L. Li, Q. Tang, Y. Yu, and S. Li. Link adaptation for spatial modulation with limited feedback. IEEE Transactions on Vehicular Technology, 61(8):3808-3813, Oct 2012.

[23] R. Rajashekar, K. V. S. Hari, and L. Hanzo. Antenna selection in spatial modulation systems. IEEE Communications Letters, 17(3):521524, March 2013

[24] K. Ntontin, M. Di Renzo, A. I. Perez-Neira, and C. Verikoukis. A lowcomplexity method for antenna selection in spatial modulation systems. IEEE Communications Letters, 17(12):2312-2315, December 2013.

[25] P. Yang, Y. Xiao, Y. L. Guan, S. Li, and L. Hanzo. Transmit antenna selection for multiple-input multiple-output spatial modulation systems IEEE Transactions on Communications, 64(5):2035-2048, May 2016.

[26] Y. He, S. Atapattu, C. Tellambura, and J. S. Evans. Opportunistic group antenna selection in spatial modulation systems. IEEE Transactions on Communications, 66(11):5317-5331, Nov 2018.

[27] R. Rajashekar, L. Yang, K. V. S. Hari, and L. Hanzo. Transmit antenna subset selection in generalized spatial modulation systems. IEEE Transactions on Vehicular Technology, 68(2):1979-1983, Feb 2019.

[28] M. . Alouini and A. J. Goldsmith. A unified approach for calculating error rates of linearly modulated signals over generalized fading channels. IEEE Transactions on Communications, 47(9):1324-1334, Sep. 1999.

[29] John G Proakis and Masoud Salehi. Digital communications, volume 4 McGraw-hill New York, 2001.

[30] Shuping Dang, Guoqing Ma, Basem Shihada, and Mohamed-Slim Alouini. Enhanced orthogonal frequency-division multiplexing with subcarrier number modulation. IEEE Internet of Things Journal, 6(5):7907$7920,2019$.

[31] Jehad M Hamamreh, Ertugrul Basar, and Huseyin Arslan. Ofdmsubcarrier index selection for enhancing security and reliability of $5 \mathrm{~g}$ urllc services. IEEE Access, 5:25863-25875, 2017.

\section{APPENDIX A COMPARISON BETWEEN ANM AND SM}

Based on the relevant available literature on adaptive spatial modulation with transmit antenna selection, we found out that for these adaptive spatial modulation schemes to work properly, a very critical assumption must be satisfied, which is that the number of available antennas at the transmitter must be greater than the number of antennas used by spatial modulation in order to create extra degree of freedom in the space domain that can help make the scheme adaptive through using channel-based transmit antenna selection. However, our proposed scheme does not require having such a critical assumption because of the inherent property that antenna number modulation brings, where indices of active antennas do not matter in MIMO-SNM, whereas number does; thus, providing a flexibility to activate antennas according to their channel qualities. Therefore, when both schemes MIMO-SM and MIMO-ANM use the same number of transmit antenna, channel based adaptive activation will only be possible with MIMO-ANM, but not MIMO-SM, unless the number of transmit antennas is increased to create extra degree of freedom in the case of MIMO-SM. Because of the above reasons along with the ones given in the response Appendix.B., and the antenna structure differences of both 
systems, a comparison between ANM-AAS and SM-AAS is not available in a logical fair manner.

We the authors including JEHAD HAMAMREH and NAOKI ISHIKAWA discussed this issue among us deeply and reached to the conclusion that you cannot make adaptive transmit antenna selection with MIMO-SM when the number of antennas at the transmitter is the same as the number of antennas used for spatial modulation purpose. This conclusion coincide with what the authors (Prof. Hanzo group) in [27] showed in their transaction paper, where zero performance gain is demonstrated when number of antennas at the transmitter is equal to the number of antennas used for spatial modulation purpose.

\section{APPENDIX B WHY ANTENNA SELECTION PROTOCOLS OF ANM AND SM ARE DIFFERENT AND WHY ANM SUPPORTS BOTH CHANNEL AND DATA DEPENDENT WHILE SM DOES NOT.}

It's possible (as it has already mentioned in Section III) to use subset antenna selection, where $K<N$ are selected from $N$ antennas, and then implement spatial modulation (SM) on the selected $K($ not $N)$ antennas only, thus SM can be made channel and data dependent in a partial manner (i.e., not all the available antennas $(N)$ are used by spatial modulation, which uses only $K<N$ antennas to modulate spatial domain indices by the transmitted data bits). In SM, you cannot first use $N$ for spatial modulation, and then activate the antennas corresponding to the best channels among $N$ antennas. However, in ANM, all the available antennas at the transmitter $(N)$ are first used for ANM, and simultaneously also used to activate the antennas corresponding to the highest channel gains.

\section{APPENDIX C WORKING PRINCIPLE OF SEQUENTIAL DETECTOR}

Sequential detector is the Maximum-Likelihood (ML) detector, which is widely accepted in the literature for a long time, but instead of applying it to detect the active antenna state and constellation symbol jointly, it detects them individually and separately; so first the number of active antennas is detected, then the symbol is detected. The sequential detector can greatly reduce the complexity at the receiver because it reduces the exhaustive search space for finding the closest active state or symbol. What we did in (7) and (8) was just an adaptation of the ML detector into our ANM scheme. In (7), the $x$ value represents the transmitted symbols which are modulated by BPSK. To decide which bits will be modulated by BPSK and which bits will be modulated by antenna numbers, Fig. 2 along with the given example in Section II.A can be a good reference for understanding the modulation process. In (8), we decide to the antenna activation by deploying the channel coefficients, namely $h_{1 i}$. As the symbols in ANM Bits group change (refer to Fig. 2 for seeing and specifying the difference between Main Bits group and ANM Bits group), the channel activation varies accordingly. This channel-based activation process is also given in Table I and Table II.

\section{APPENDIX D THE REASON WHY THE BER RESULTS DO NOT SHOW LOWER VALUES THAN $10^{-4}$}

The reason why we did not show the results regarding to lower BER values such as $10^{-5}$ or $10^{-6}$ in the figures can be explained with two reasons. The first one is because the considered range of SNR values is less than $30 \mathrm{~dB}$, which is close to most practical wireless scenarios, where the SNR is usually between 0 and $30 \mathrm{~dB}$. Thus, if we were to simulate the system till 50 or $60 \mathrm{~dB}$, which is usually not very practical, then we would reach $10^{-5}$ or $10^{-6}$ BER results. The second reason why we don't exhibit BER results reaching to $10^{-5}$ or $10^{-6}$ is because we are simulating a MISO system with only one antenna at the receiver, which means there is no receive diversity gain, and thus the BER results are not good enough to hit those values at low SNRs, so there is nothing to show at $10^{-5}$ or $10^{-6}$.

\section{APPENDIX E DIVERSITY GAIN IN MIMO-ANM}

It should be clearly stated that the diversity gain is produced by the prevention of using 'bad' antenna(s), which corresponds to channels with low power gain, through using the proposed channel-based look-up mapping table. It's also obvious from the simulation results that the proposed MIMOANM-AAS greatly affects the error probability versus SNR curve in such a way that asymptotically, in the limit of large SNR, the error probability of MIMO-ANM and MIMOANM-AAS performance curves become parallel and as such the difference in SNR (horizontal separation) between these curves approaches a fixed finite amount. This asymptotic SNR separation is what is commonly referred to as coding gain. Mathematically, if asymptotically the error probability behaves as $P(E) \cong\left(G_{c} \gamma\right)^{-G_{d}}$, then in the limit of large SNR, the coding gain $G_{c}$ represents the horizontal shift in the error probability performance relative to the benchmark curve $P(E) \cong \gamma^{\left(-G_{d}\right)}$. It is also observed that the error performance of MIMO systems is dominated by the worst active antenna with the lowest channel power gain over all legitimate antenna activation patterns (AAP). That is, the diversity gain in the space domain is produced by the prevention of using 'bad' antenna(s) in the look-up mapping table. [30], [31]. 


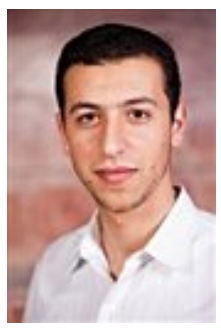

JEHAD M. HAMAMREH received the B.Sc. degree in electrical and telecommunication engineering from An-Najah University, Nablus, in 2013, and the Ph.D. degree in electrical electronics engineering and cyber systems from Istanbul Medipol University, Turkey, in 2018. He was a Researcher with the Department of Electrical and Computer Engineering, Texas A\&M University. $\mathrm{He}$ is currently an Assistant Professor with the Electrical and Electronics Engineering Department, Antalya International (Bilim) University, Turkey. His current research interests include wireless physical and MAC layers security, orthogonal frequency-division multiplexing multiple-input multiple-output systems, advanced waveforms design, multi-dimensional modulation techniques, and orthogonal/non-orthogonal multiple access schemes for future wireless systems. He is a Regular Reviewer for various peer-reviewed journals as well as a TPC Member for several international conferences. He is very open for collaboration and can be reached via email at jehad.hamamreh@gmail.com.

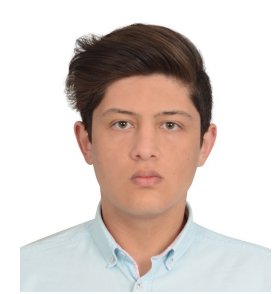

MUHAMMET KIRIK He is a senior electrical and electronics engineering student at Antalya International (Bilim) University, Turkey. His current research interests include orthogonal frequencydivision multiplexing multiple-input multipleoutput systems, multi-dimensional modulation techniques, and orthogonal/non-orthogonal multiple access schemes for future wireless systems.

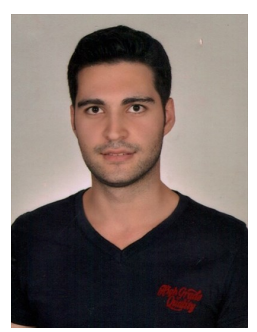

MEHMET O. SAGMAN He is a senior electrical and electronics engineering student at Antalya International (Bilim) University, Turkey. His current research interests include orthogonal frequencydivision multiplexing multiple-input multipleoutput systems, multi-dimensional modulation techniques, and orthogonal/non-orthogonal multiple access schemes for future wireless systems.

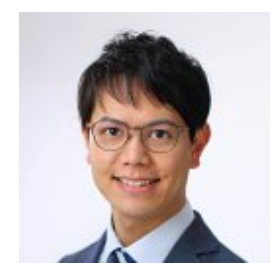

NAOKI ISHIKAWA (S'13-M'17) was born in Kanagawa, Japan, in 1991. He received the B.E., M.E., and Ph.D. degrees from the Tokyo University of Agriculture and Technology, Tokyo, Japan, in 2014, 2015, and 2017, respectively. From June 2015 to September 2015, he was an Academic Visitor with the School of Electronics and Computer Science, University of Southampton, U.K. From April 2016 to March 2017, he was a Research Fellow of the Japan Society for the Promotion of Science. From April 2017 to 2020, he was an Assistant Professor with the Graduate School of Information Sciences, Hiroshima City University, Japan. Since April 2020, he has been an associate Professor with the Graduate School of Information Sciences, Yokohama National University, Japan. Dr. Ishikawa received eight domestic awards, including the Yasujiro Niwa Outstanding Paper Award from Tokyo Denki University, in 2018, the Telecom System Technology Student Award (honorable mention) from Telecommunications Advancement Foundation of Japan, in 2014, and the Outstanding Paper Award for Young CC Researchers from NEC CC Foundation, in 2014. He was certified as an Exemplary Reviewer of the IEEE Transactions on Communications, in 2017. 